\title{
Modified Finite-Difference Beam Propagation Method Based on the Generalized Douglas Scheme for Variable Coefficients
}

\author{
Junji Yamauchi, Member, IEEE, Jun Shibayama, and Hisamatsu Nakano, Fellow, IEEE
}

\begin{abstract}
The accuracy of the implicit finite-difference beam propagation method (FD-BPM), in which the phase term is not split, is improved using the generalized Douglas scheme. The propagation error of the fundamental mode in two- and three-dimensional waveguides is evaluated by the mode-mismatch loss calculation. It is demonstrated that the truncation error is reduced to $O(\Delta x)^{4}$ in the transverse direction, even when the parabolic wave equation contains variable coefficients. The computational time is almost identical to the conventional FDBPM based on the Crank-Nicholson scheme.
\end{abstract}

\section{INTRODUCTION}

$\mathbf{T}$ THE ACCURACY of the finite difference beampropagation method (FD-BPM) [1] can be improved by replacing the Crank-Nicholson $(\mathrm{CN})$ scheme with the Douglas scheme [2]. The truncation error of $\mathrm{O}(\Delta x)^{2}$ in the transverse direction can be reduced to $\mathrm{O}(\Delta x)^{4}$. Yevick et al. [3] first introduced the Douglas scheme into the propagating beam analysis with splitting the phase term. This procedure, however, has a disadvantage that the step length $\Delta z$ in the propagation direction should be small. Recently, Sun and Yip [4] formulated the modified FD-BPM based on the Douglas scheme in which the phase term is not split. Their formulation has an advantage that the numerical result is not a sensitive function of $\Delta z$. However, they employed the Douglas scheme for constant coefficients, so that they did not take into account variable coefficients in the phase term. This does not ensure the reduction of the truncation error to $\mathrm{O}(\Delta x)^{4}$, when the space has the index change in the transverse directions.

In this letter, we present a modified FD-BPM, based on the Douglas scheme generalized for variable coefficients. The accuracy of our formulation is better than that in [4] and the truncation error of $\mathrm{O}(\Delta x)^{4}$ is ensured. The present scheme is also applied to the alternating direction implicit method (ADIM) [5], [6] for the analysis of a three-dimensional optical waveguide.

\section{Discussion}

For simplicity, we explain the present scheme using a twodimensional problem. When the truncation error is included, the second derivative of function $E$ is expressed as

$$
\frac{\partial^{2} E}{\partial x^{2}}=\frac{\delta^{2} E}{\Delta x^{2}}-\frac{1}{12} \frac{\partial^{4} E}{\partial x^{4}} \Delta x^{2}+O(\Delta x)^{4}
$$

Manuscript received September 27, 1994; revised November 23, 1994. The authors are with the College of Engineering, Hosei University, 3-7-2, Kajino-cho, Koganei, Tokyo 184, Japan.

IEEE Log Number 9411272. where $\delta^{2} E=E_{i+1}-2 E_{i}+E_{i-1}$ is defined. In the conventional FD-BPM, only the first term in the right-hand side of (1) is evaluated, so that the truncation error is $\mathrm{O}(\Delta x)^{2}$. Higher accuracy can be realized by evaluating the second term in (1).

The parabolic wave equation is expressed as

$$
\sigma \frac{\partial E}{\partial z}=\frac{\partial^{2} E}{\partial x^{2}}+\nu E
$$

where $\sigma=2 j k n_{0}$ and $\nu=k^{2}\left[n(x, z)^{2}-n_{0}^{2}\right]$, in which $k$ is the free space wavenumber, $n(x, z)$ is the index profile of the waveguide and $n_{0}$ is the reference index to be appropriately chosen. We substitute (2) into (1), replacing $\partial^{4} E / \partial x^{4}$ with $\delta^{2}(\sigma \partial E / \partial z-\nu E) / \Delta x^{2}+O(\Delta x)^{2}$. Then, the following difference equation can be derived.

$$
\begin{aligned}
\frac{\delta^{2} E}{\Delta x^{2}}= & \frac{1}{12}\left(\sigma \frac{\partial E}{\partial z}\right)_{i+1}+\frac{5}{6}\left(\sigma \frac{\partial E}{\partial z}\right)_{i}+\frac{1}{12}\left(\sigma \frac{\partial E}{\partial z}\right)_{i-1} \\
& -\frac{1}{12}(\nu E)_{i+1}-\frac{5}{6}(\nu E)_{i}-\frac{1}{12}(\nu E)_{i-1}+O(\Delta x)^{4}
\end{aligned}
$$

From (3), we can easily obtain the six-point scheme with centered $z$ difference. It should be noted that the variable terms $\nu_{i \pm 1}$ appear, which are not taken into account in [4]. These terms are significant for the reduction in the truncation error, as will be seen later.

Since (3) leads to a tridiagonal system of linear equations, an efficient computing algorithm can be used as in the case of the $\mathrm{CN}$ scheme. It follows that the computational speed is almost identical to that in the conventional FD-BPM.

As a measure of the numerical accuracy, we evaluate the mode-mismatch loss $L_{M}$, which is known to be a sensitive indicator of the accuracy of the BPM [1]. In a two-dimensional waveguide, $L_{M}$ is defined as

$$
L_{M}=-10 \log \left[\left|\int E_{0} E^{*} d x\right|^{2} /\left[\int\left|E_{0}\right|^{2} d x\right]^{2}\right][\mathrm{dB}]
$$

where $E$ is the propagating field and $E_{0}$ is the incident field of the fundamental mode.

In the following analysis, the transparent boundary condition [7] is imposed at the edge of the computational window, and the reference index is chosen to be that in the cladding. 


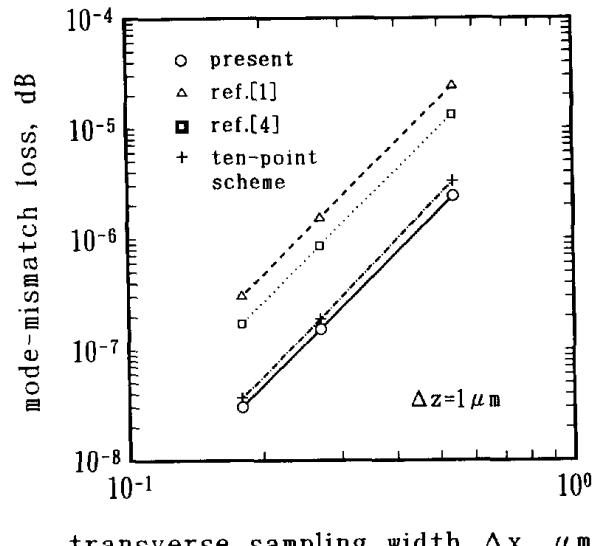

Fig. 1. Mode-mismatch loss as a function of transverse sampling width $\Delta x$. A step-index slab waveguide is investigated. Four schemes are compared. The least loss is observed in the generalized Douglas scheme.

We first investigate the propagation error in a step-index slab waveguide whose core width is $2 D=7.546 \mu \mathrm{m}$. Fig. 1 shows the mode-mismatch loss as a function of transverse sampling width. The refractive indices of the core and cladding are $N_{C O}=1.002$ and $N_{C L}=1.000$, respectively. The computational window dimension is $107.8 \mu \mathrm{m}$ and the wavelength is $\lambda=1 \mu \mathrm{m}$. The loss evaluation is carried out at a propagation distance of $500 \mu \mathrm{m}$. For reference, the results obtained by the $\mathrm{CN}$ scheme in [1] and by the scheme in [4] are also shown. It is found that the mode-mismatch loss observed for the present scheme is smaller than those for the previous ones. Comparison between the result in the present scheme and that in [4] indicates that the inclusion of the variable terms $\nu_{i \pm 1}$ remarkably improves the accuracy.

To confirm the accuracy of the present scheme, we have also tested the FD-BPM using a higher order (ten-point) scheme, since its truncation error is $\mathrm{O}(\Delta x)^{4}$. Fig. 1 indicates that the accuracy is close to that of the present scheme. It should be noted, however, that the ten-point scheme results in computational penalty due to the solution of the pentadiagonal matrix.

One may feel strange that the ten-point scheme is slightly less accurate than the present scheme. This is, however, due to the fact that in the ten-point scheme the centered difference formula is again used to evaluate $\partial^{2} E / \partial x^{2}$ in $\partial^{4} E / \partial x^{4}=$ $\delta^{2}\left(\partial^{2} E / \partial x^{2}\right) / \Delta x^{2}$, while $\partial^{2} E / \partial x^{2}$ is exactly replaced in the present scheme.

Fig. 2 shows the mode-mismatch loss as a function of propagation step length $\Delta z$. The computation parameters which are not specified are the same as those in Fig. 1. The results for the present scheme are compared with those obtained from the $\mathrm{CN}$ scheme, without splitting the phase term in [1], and the Douglas scheme, with splitting the phase term in [3]. It is worth mentioning that the loss for the present scheme is not a sensitive function of $\Delta z$, as in the case of the $\mathrm{CN}$ scheme [8]. In contrast, the loss observed for the scheme with splitting the phase term increases as $\Delta z$ is increased.

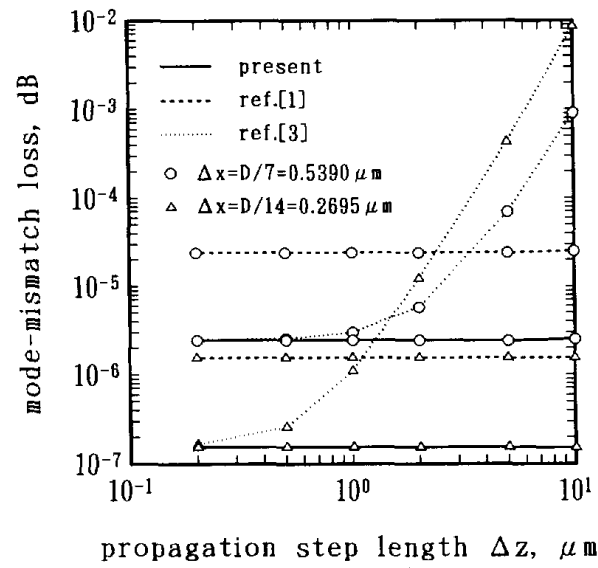

Fig. 2. Mode-mismatch loss as a function of propagation step length $\Delta z$. The waveguide is the same as that in Fig. 1. The cases with and without splitting the phase term are compared. The results obtained from the generalized Douglas scheme without splitting the phase term are not sensitive to the change in the propagation step length, as in the case of the Crank-Nicholson scheme without splitting the phase term.

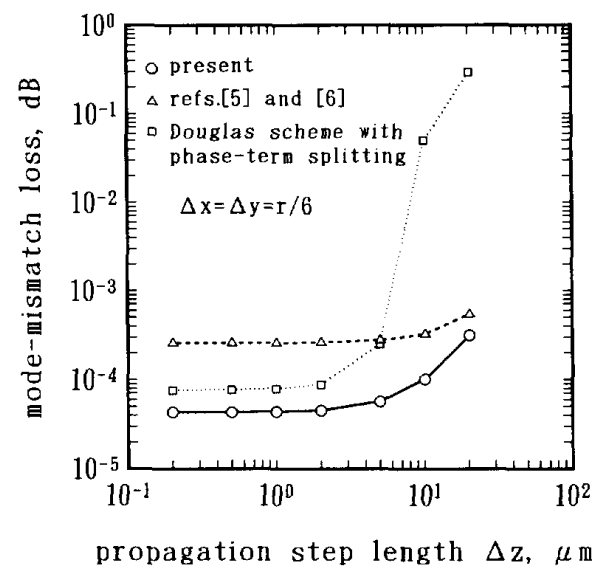

Fig. 3. Mode-mismatch loss as a function of propagation step length $\Delta z$. A step-index fiber is investigated. The least loss is found in the ADIM based on the generalized Douglas scheme without splitting the phase term.

As a further test on the accuracy, we calculate the coupling length when the same waveguides are placed with a gap of $2 D$. The result is compared with that obtained from the exact solution. For example, for $\Delta x=D / 5=0.7546 \mu \mathrm{m}$, the present scheme exhibits an error of less than $0.13 \%$, while the $\mathrm{CN}$ scheme does $0.21 \%$, indicating the substantial improvement in the accuracy.

We next apply the present scheme to the Peaceman-Rachford ADIM. The waveguide considered here is a step-index fiber with a core radius of $r=5 \mu \mathrm{m}$. The refractive indexes are chosen to be $N_{C O}=1.504$ and $N_{C L}=1.500$. The wavelength of $\lambda=1.55 \mu \mathrm{m}$ is used. The computation window dimensions are given by $L_{x} \times L_{y}=125$ $\times 125 \mu \mathrm{m}^{2}$. Fig. 3 shows the mode-mismatch loss evaluated at $z=500 \mu \mathrm{m}$ as a function of $\Delta z$. It is clear that the 
accuracy of the present scheme is improved, when compared with the ADIM's based on the CN scheme [5], [6] and on the Douglas scheme with phase-term splitting.

\section{CONCLUSION}

The modified FD-BPM, based on the generalized Douglas scheme in which the phase term is not split, has been formulated. The truncation error is reduced to $\mathrm{O}(\Delta x)^{4}$ in the transverse direction, even when the parabolic wave equation contains variable coefficients. The computation time is almost identical to the conventional FD-BPM based on the Crank-Nicholson scheme. We also apply the present scheme to the alternating direction implicit method and demonstrate its effectiveness in the analysis of a three-dimensional waveguide.

\section{ACKNOWLEDGMENT}

The authors wish to thank $T$. Iwata, who investigated the FD-BPM based on the ten-point scheme.

\section{REFERENCES}

[1] Y. Chung and N. Dagli, "An assessment of finite difference beam propagation method," IEEE J. Quantum, Electron., vol. 26, no. 8, pp. $1335-1339,1990$.

[2] A. R. Mitchell and D. F. Griffiths, The Finite Difference Method in Partial Differential Equations. New York: Wiley, 1980, ch. 2

[3] D. Yevick, B. Hermansson, and M. Glasner, "Fresnel and wide-angle equation analyses of microlenses," IEEE Photon. Technol. Lett., vol. 2, no. 6, pp. $412-414,1990$.

[4] L. Sun and G. L. Yip, "Modified finite-difference beam-propagation method based on the Douglas scheme," Opt. Lett., vol. 18, no. 15, pp. $1229-1231,1993$.

[5] J. Yamauchi, T. Ando, and H. Nakano, "Beam-propagation analysis of optical fibers by alternating direction implicit method," Electron. Lett., vol. 27 , no. 18 , pp. 1663-1665, 1991.

[6] P. L. Liu and B. J. Li, "Semivectorial beam-propagation method for analyzing polarized modes of rib waveguides," IEEE J. Quantum Electron., vol. 28, no. 4, pp. 778-782, 1992.

[7] G. R. Hadley, "Transparent boundary condition for beam propagation," Opt. Lett., vol. 16, no. 9, pp. 624-626, 1991.

[8] D. Yevick and B. Hermansson, "Efficient beam propagation techniques," IEEE J. Quantum Electron., vol. 26, no. 1, pp. 109-112, 1990. 